Research Paper

\title{
Exosomes from Human Synovial-Derived Mesenchymal Stem Cells Prevent Glucocorticoid-Induced Osteonecrosis of the Femoral Head in the Rat
}

\author{
Shang-Chun Guo ${ }^{*}$, Shi-Cong Tao ${ }^{2 *}$, Wen-Jing Yin², Xin Qi ${ }^{2}$, Jia-Gen Sheng ${ }^{2 \bowtie}$, Chang-Qing Zhang ${ }^{1,2 \bowtie}$ \\ 1. Institute of Microsurgery on Extremities, Shanghai Jiao Tong University Affiliated Sixth People's Hospital, 600 Yishan Road, Shanghai 200233, China; \\ 2. Department of Orthopedic Surgery, Shanghai Jiao Tong University Affiliated Sixth People's Hospital, 600 Yishan Road, Shanghai 200233, China. \\ ${ }^{*}$ Co-first authors: These authors contributed equally to this work.
}

$\triangle$ Corresponding authors: Chang-qing Zhang, Ph.D., M.D. Department of Orthopedic Surgery, Shanghai Jiao Tong University Affiliated Sixth People's Hospital, Shanghai 200233, China. E-mail: zhangcq@sjtu.edu.cn Or Jia-Gen Sheng, Ph.D., M.D. Department of Orthopedic Surgery, Shanghai Jiao Tong University Affiliated Sixth People's Hospital, Shanghai 200233, China. E-mail: jiagen_sheng@163.com.

() Ivyspring International Publisher. Reproduction is permitted for personal, noncommercial use, provided that the article is in whole, unmodified, and properly cited. See http://ivyspring.com/terms for terms and conditions.

Received: 2016.05.13; Accepted: 2016.08.19; Published: 2016.10.17

\begin{abstract}
Osteonecrosis of the femoral head (ONFH) represents a debilitating complication following glucocorticoid (GC)-based therapy. Synovial-derived mesenchymal stem cells (SMSCs) can exert protective effect in the animal model of GC-induced ONFH by inducing cell proliferation and preventing cell apoptosis. Recent studies indicate the transplanted cells exert therapeutic effects primarily via a paracrine mechanism and exosomes are an important paracrine factor that can be directly used as therapeutic agents for tissue engineering. Herein, we provided the first demonstration that the early treatment of exosomes secreted by human synovial-derived mesenchymal stem cells (SMSC-Exos) could prevent GC-induced ONFH in the rat model. Using a series of in vitro functional assays, we found that SMSC-Exos could be internalized into bone marrow derived stromal cells (BMSCs) and enhance their proliferation and have anti-apoptotic abilities. Finally, SMSC-Exos may be promising for preventing GC-induced ONFH.
\end{abstract}

Key words: osteonecrosis of the femoral head; glucocorticoid; synovial-derived mesenchymal stem cells; apoptosis.

\section{Introduction}

Osteonecrosis of the femoral head (ONFH) is a disabling and progressive disease commonly induced by high-dose and (or) long-term administration of steroid hormones [1-3]. Promotion of apoptosis of bone marrow cells is thought to be an important reason for causing $\mathrm{ONFH}$, as it plays a crucial role in destructing the formation of bone tissues [4-10]. Thus, strategies to prevent apoptosis and promote proliferation of bone marrow cells at the early phase may dramatically trigger the repairing process and halt the progression of GC-associated ONFH.

In recent years, cell-based therapy has emerged as a promising strategy for bone tissue regenerative treatments [11, 12]. Mesenchymal stem cells (MSCs) have been isolated from a variety of tissues including adipose tissue [13], bone marrow (BM) [14], and synovium membrane [15]. Synovial-derived mesenchymal stem cells (SMSCs) possessed high self-renewal ability over at least 10 passages without obvious senescence and can be induced to differentiate toward chondrogenesis, osteogenesis, myogenesis and adipogenesis, independently of donor age [15]. However, the direct use of stem cells remains limited by issues of potential chromosomal variation, immunological rejection, thrombosis, etc [16-18]. Hence, it is necessary to develop a novel strategy that can fully exert the therapeutic effects of stem cells and avoid the risks associated with the direct use of them.

Exosomes are 40-150 nm-sized nanoparticles 
originating from multivesicular bodies (MVBs) and function as important paracrine effectors in intercellular communication by transferring proteins and genetic materials to target cells [19]. It has been shown that exosomes exhibit similar functional properties to the cells from which they are derived and direct treatment with these nanoparticles has no apparent adverse effects such as vascular obstructive risks, immunogenicity, and tumorigenicity [20, 21]. Currently, there are no reports regarding application of exosomes in GC-induced ONFH. In consideration of the self-renewal ability and multi-differential potentialities of SMSCs, we hypothesized that their released exosomes (SMSC-Exos) could exert protective effects on GC-induced ONFH.

In the present study, we used the human synovial-derived mesenchymal stem cells (SMSCs) as a "factory" to generate exosomes and investigated whether the early administration of these exosomes was able to prevent GC-induced ONFH in the rat model. The results showed that SMSC-Exos effectively attenuated a series of GC-induced ONFH-related responses, such as the suppression of cell proliferation and cell apoptosis, bone mineral loss, and fat tissue accumulation. We evaluated the effects of SMSC-Exos on the behavior of bone marrow derived stromal cells (BMSCs) in vitro and found that SMSC-Exos could be internalized into BMSCs and enhance BMSCs' proliferation, and resistance to serum deprivation-induced apoptosis. These data suggest that SMSC-Exos may enhance the proliferation and anti-apoptotic responses of bone marrow cells, then facilitate tissue regeneration and prevent GC-induced ONFH.

\section{Materials and Methods}

\subsection{Isolation of human synovial-derived mesenchymal stem cells}

Human synovial membrane samples (wet weight 20-50 mg) were obtained aseptically from arthroscopically assisted treatment with permission from the patients and the Institutional Review Board at Shanghai Sixth People's Hospital. Synovial membrane samples were rinsed three times with Dulbecco's Phosphate Buffered Saline (DPBS, Corning) supplemented with penicillin-streptomycin solution (PS; 100 units/ml penicillin, $100 \mu \mathrm{g} / \mathrm{ml}$ streptomycin, Gibco), minced carefully, and digested with $0.2 \%$ type I collagenase (Life Technologies) in high-glucose Dulbecco's modified Eagle's medium (high-glucose DMEM; Hyclone) with 10\% fetal bovine serum (FBS; Gibco). Cells were incubation at $37^{\circ} \mathrm{C}$ overnight, before cells were collected by centrifugation, washed three times, resuspended in high-glucose DMEM supplemented with 10\% FBS and PS (100 units/ml penicillin, $100 \mu \mathrm{g} / \mathrm{ml}$ streptomycin). Resuspended cells were plated in a T25 culture flask and incubated to attach for 4 days. After changing the medium to remove non-adherent cells, the medium was replaced every 3 days. Cells were cultured in monolayer in high-glucose DMEM supplemented with $10 \%$ FBS and PS (100 units/ml penicillin, $100 \mathrm{\mu g} / \mathrm{ml}$ streptomycin) at $37{ }^{\circ} \mathrm{C}$ in humidified atmosphere of 5\% CO2.

\subsection{Characterization of human synovial-derived mesenchymal stem cells}

Cells were blocked with 3\% BSA for 30 min and then incubated with the following primary antibodies (BD Biosciences, San Jose, CA, USA) for one hour at room temperature: phycoerythrin (PE)-conjugated anti-CD44, PE-conjugated anti-CD73, allophycocyanin (APC)-conjugated anti-CD34, fluorescein isothiocyanate (FITC)-conjugated anti-CD45. Nonspecific fluorescence was determined by incubation of similar cell aliquots with isotype-matched mouse monoclonal antibodies (BD Biosciences). Cells were analyzed by the Guava easyCyte $^{\mathrm{TM}}$ Flow Cytometer (Millipore, Billerica, MA).

The differentiative capacity of SMSCs into osteogenic, adipogenic and chondrogenic lineages was tested using specific differentiation medium (Cyagen).

\subsection{Isolation and identification of exosomes derived from human synovial-derived mesenchymal stem cells}

After reaching about $80 \%$ confluency, SMSCs were rinsed with PBS and cultured in MesenGro hMSC Medium (StemRD) deprived of FBS for 48 hours. The conditioned media $(\mathrm{CM})$ of SMSCs was obtained and centrifuged at $300 \times \mathrm{g}$ for $10 \mathrm{~min}$ and $2000 \times \mathrm{g}$ for $10 \mathrm{~min}$ to remove dead cells and cellular debris. The supernatant was filtered using a $0.22 \mu \mathrm{m}$ filter (Millipore) and centrifuged at $4000 \times \mathrm{g}$ to about $200 \mu \mathrm{L}$ by ultra-filtration in a $15 \mathrm{~mL}$ Amicon Ultra- 15 Centrifugal Filter Unit (Millipore). The ultrafiltration liquid was washed twice with PBS and re-ultrafiltrated at $4000 \times \mathrm{g}$ to $200 \mu \mathrm{L}$. For exosomes purification, the liquid was overlaid onto $30 \%$ sucrose- $\mathrm{D}_{2} \mathrm{O}$ cushion in a sterile Ultra-Clear ${ }^{\mathrm{TM}}$ tube (Beckman Coulter, Brea, CA) and ultracentrifuged at $100000 \times \mathrm{g}$ for 1 hour. The pelleted exosomes were resuspended in PBS and centrifuged at $4000 \times \mathrm{g}$ to about $200 \mu \mathrm{L}$. All procedures were performed at $4{ }^{\circ} \mathrm{C}$. Exosomes were stored at $-80{ }^{\circ} \mathrm{C}$ or used for downstream experiments.

Dynamic light scattering (DLS) analysis, 
transmission electron microscopy (TEM), and western blotting were used to identify the collected exosomes. The size distribution of exosomes was measured by DLS analysis using Nanosizer ${ }^{\mathrm{TM}}$ technology (Malvern). Samples were diluted 1000-fold with filtered DPBS. Data processing and analysis were carried out on the Zetasizer software (Malvern). The morphology of exosomes was observed by TEM. Exosomes were loaded on a continuous carbon grid and visualized by a Hitachi H-7650 transmission electron microscope (Hitachi, Tokyo, Japan). The exosomal surface markers including CD9, CD63, CD81, and tumor susceptibility gene TSG101, and were analyzed by western blotting. The procedures were detailed in Section 2.6. The MesenGro hMSC Medium was ultrafiltrated as mentioned above and the ultrafiltration liquid was used as negative control (NC) in western blot.

\subsection{In vivo effects of SMSC-Exos on GC-induced ONFH rats}

\subsubsection{Animal model and grouping}

All experimental and animal care procedures were approved by Animal Research Ethics Committee of Shanghai Sixth People's Hospital and performed in accordance with the guidelines of the National Institutes of Health Guidelines for the Care and Use of Laboratory Animals. Sixty 8-week-old healthy female Sprague-Dawley (SD) rats weighing 200-210 gram were used in this study. The rats were randomly and averagely divided into three groups: (1) normal group (served as controls and did not receive any treatment) $(\mathrm{n}=20)$; (2) model group (rats with GC-induced ONFH) (n=20); (3) SMSC-Exos group (rats with GC-induced ONFH treated with SMSC-Exos) $(n=20)$. Each group was divided into two subgroups, short-term subgroup $(\mathrm{n}=5)$ and long-term subgroup $(n=15)$. In long-term subgroup, to induce ONFH of the rats, methylprednisolone (MPS; Pfizer, USA) (20 $\mathrm{mg} / \mathrm{kg} / \mathrm{d}$ ) was intramuscularly injected into rats, on the first three days of every week, for three weeks. In short-term subgroup, MPS was only injected on the first three days. Every time after MPS injection, rats in the SMSC-Exos group and model group were respectively administered $1 \times 10^{11}$ particles of exosomes (dissolved in $200 \mu \mathrm{L}$ of PBS) or an equal volume of PBS via the vein of tail. In short-term subgroup, after three-day injection, the five rats in each group were anesthetized by an intraperitoneal injection of $400 \mathrm{mg} / \mathrm{kg}$ chloral hydrate and the femoral heads were collected for TUNEL assay and ki67 immunostaining. Six weeks later, the rats in long-term subgroup were anesthetized and the femoral heads were assessed by micro-CT, immunohistochemical and histological analyses. In all groups, none of the rats died before these assessments. No antibiotic agents were used throughout the study.

\subsubsection{Micro-CT analysis}

The femoral heads were dissected from rats, fixed overnight in formalin and analyzed by SkyScan1178 (Bruker MicroCT, Kontich, Belgium). The scanner was set at a resolution of $9 \mu \mathrm{m}$ per pixel. The trabecular bone was segmented from bone marrow and analyzed to determine trabecular thickness (Tb.Th), trabecular separation (Tb.Sp), bone volume per tissue volume (BV/TV), and trabecular number (Tb.N). Three planes (coronal section, sagittal section and transverse section) of representative samples of each group were generated using DataViewer (Bruker MicroCT).

\subsubsection{Histological and immunohistochemical analyses}

The femoral heads were excised and fixed in $10 \%$ buffered formalin solution for 24 hours, decalcified in $10 \%$ EDTA for 28 days, and embedded in paraffin. Samples were cut into 5 - $\mu \mathrm{m}$ sections, deparaffinized in xylene, rehydrated in a graded series of ethanol, and rinsed in distilled water. Hematoxylin and eosin (H\&E) staining was performed for histological observations. The extent of osteogenesis was assessed by immunohistochemical (IHC) analysis of osteocalcin $(\mathrm{OCN})$, respectively. Images were acquired with a fluorescence microscope (IX81; Olympus, Hamburg, Germany). Antibodies were obtained from Abcam (Cambridge, Cambs, Britain).

\subsubsection{TUNEL assay and ki67 immunostaining}

The femoral heads were dissected and fixed overnight in $4 \%$ paraformaldehyde, decalcified in $10 \%$ EDTA for 28 days, and embedded in paraffin. Samples were cut into $5-\mu \mathrm{m}$ sections, deparaffinised in xylene, and rehydrated with a graded ethanol series. TUNEL staining kit (Roche, Nutley, NJ) were used to detect DNA strand breaks according to the manufacturer' instructions. The proliferation of cells was immunostained with Ki67 antibody (Abcam, Cambridge, Cambs, Britain). Nuclei was stained with DAPI. Images were acquired with a LSM-880 confocal-microscopes (Carl Zeiss; Germany). The number of TUNEL-positive cells (apoptotic cells) and Ki67-positive cells (proliferative cells) per field was evaluated in five fields per section and five sections per femoral head.

\subsection{In vitro effects of SMSC-Exos on bone marrow derived stromal cells}

\subsubsection{Cells culture and treatments}

BMSCs were isolated from healthy donors (age, 
30-50 years old) who underwent amputation due to severe trauma with permission from the patients and the Institutional Review Board at Shanghai Sixth People's Hospital. Briefly, cancellous bone was obtained during the operation and was first flushed with a-MEM (Hyclone) with 10\% FBS (Gibco) and then transferred to a T75 culture flasks. BMSCs were isolated by adherence and were cultured in a-MEM media containing $10 \%$ FBS. BMSCs were maintained at $37^{\circ} \mathrm{C}$ in a moist atmosphere of $21 \% \mathrm{O}_{2}, 5 \% \mathrm{CO}_{2}$, and $74 \% \mathrm{~N}_{2}$. For assessing the effects of SMSC-Exos on BMSCs' function, the cells were cultured in a-MEM media and divided into four groups: (1) control group (no treatment); (2) DEX group (treated with $50 \mu \mathrm{M}$ dexamethasone (Sigma)); (3) SMSC-Exos group (treated with $1 \times 10^{11}$ particles/mL of exosomes); (4) DEX+SMSC-Exos group (treated with $50 \mu \mathrm{M}$ DEX and $1 \times 10^{11}$ particles/mL of exosomes). Twenty-four hours later, a series of in vitro assays were performed on BMSCs.

\subsubsection{Exosomes uptake by BMSCs}

SMSCs were labeled with DiL dye (Molecular Probes, Eugene, OR) according to the manufacturer's protocol. Briefly, cells were trypsinized and resuspended in $1 \mathrm{~mL}$ of serum-free a-MEM media. 5 $\mu \mathrm{L}$ of cell-labeling solution was added to the cells' medium and incubated with cells at $37^{\circ} \mathrm{C}$ for $15 \mathrm{~min}$. The cell-labeled suspension was centrifuged at $300 \times \mathrm{g}$ for $15 \mathrm{~min}$ and the supernatant was discarded. Cells were washed with PBS and cultured in MesenGro hMSC Medium (StemRD) for 24 hours. Exosomes were harvested and incubated with BMSCs at $37^{\circ} \mathrm{C}$ for 2 hours. BMSCs were fixed with $4 \%$ paraformaldehyde for 15 min and stained with DAPI for $5 \mathrm{~min}$. Cells were analyzed with a fluorescence microscope (Leica DMI6000B).

\subsubsection{Cells proliferation assay}

Cells proliferation was evaluated by cell counting kit-8 assay (CCK-8; Dojindo, Kumamoto, Japan). Cells ( $5 \times 10^{3}$ cells per well) were seeded onto 96-well plates. A group without cells served as the blank. At day 0,1, 3 and 7, CCK-8 solution $(10 \mu \mathrm{L}$ per well) was added to cells, followed by incubation at $37^{\circ} \mathrm{C}$ for 1 hours. The absorbance was measured at 450 $\mathrm{nm}$ by a microplate reader and the optical density values represented the survival/proliferation of cells.

\subsubsection{Cells apoptosis assay}

Apoptosis of cells was measured using the annexin V-FITC early apoptosis detection kit (Cell Signaling Technology, Danvers, MA). Briefly, after 24 hours of culture in serum-free a-MEM media, cells were harvested, washed with DPBS, and resuspended in $96 \mu \mathrm{L}$ of annexin $\mathrm{V}$ binding buffer, followed by incubation with $1 \mu \mathrm{L}$ of annexin V-FITC and $12.5 \mu \mathrm{L}$ of propidium iodide (PI) for $10 \mathrm{~min}$ on ice in the dark. Next, the cell suspension was diluted to a final volume of $250 \mu \mathrm{L}$ with annexin $\mathrm{V}$ binding buffer and analyzed by flow cytometry.

\subsection{Western blotting analysis}

Protein samples were diluted 1:5 with protein loading buffer ( $6 \times$; Transgen Biotech, Beijing, China) and heated at $95{ }^{\circ} \mathrm{C}$ for $5 \mathrm{~min}$. Protein extracts were separated on a $10 \%$ sodium dodecyl sulfate-polyacrylamide gel electrophoresis (SDS-PAGE) gel, blotted onto polyvinylidene fluoride membranes, and blocked with 5\% non-fat dried milk in TBST. The membranes were incubated with primary antibodies at $4^{\circ} \mathrm{C}$ overnight and with the horseradish peroxidase (HRP)-conjugated secondary antibodies at $37{ }^{\circ} \mathrm{C}$ for 2 hours. The primary antibodies (anti-CD9, anti-CD63, anti-TSG101, and anti-CD81) were obtained from SBI (System Biosciences; Palo Alto, CA, USA) and the secondary antibodies were obtained from Cell Signaling Technology (Danvers, MA, USA). Protein bands were visualized by enhanced chemiluminescence reagent (Thermo Fisher Scientific, Waltham, MA) and imaged by a FluorChem E gel documentation system (ProteinSimple, San Jose, CA).

\subsection{Statistical analysis}

All of these experiments were repeated for three times. The data were shown as means \pm standard deviation (SD). Means of multiple groups were compared with one-way analysis of variance (ANOVA). Independent-sample $t$ test was used to compare means between two different groups. Fisher's exact test was used to compare incidence of disease between two different groups. Statistical analysis was conducted using SPSS 20.0 (IBM, Armonk, NY). $P$ values $<0.05$ were considered statistically significant.

\section{Results}

\subsection{Characterization of human synovial-derived mesenchymal stem cells and their released exosomes (SMSC-Exos)}

SMSC colonies appeared between 7 and 21 days of culture. As viewed under the microscopy, SMSCs exhibited typical spindle-shaped morphology (Fig. 1A). Immunostaining and flow cytometry analyses showed these cells were highly positive for CD73 (mesenchymal stem cells positive markers) and CD44 (synovium-derived MSCs positive markers), but were negative for CD45 and CD34 (Fig. 1B). The potential of adipogenic differentiation was studied by measuring the formation of small cytoplasmic lipid 
granule by Oil Red $\mathrm{O}$ staining after 2 weeks of differentiation. The potential of osteogenic differentiation was studied by measuring the formation of calcium mineral deposits by Alizarin Red staining after 21 days of differentiation. And the potential of chondrogenic differentiation was studied by Alcian blue staining after 4 weeks of differentiation in alginate beads (Fig. 1C). These results suggest that the SMSCs possessed MSC properties and pluripotency. All these data unequivocally confirmed that SMSCs were successfully isolated from human synovial membrane.

Next, SMSC-Exos were isolated from the conditioned media of SMSCs by differential

A

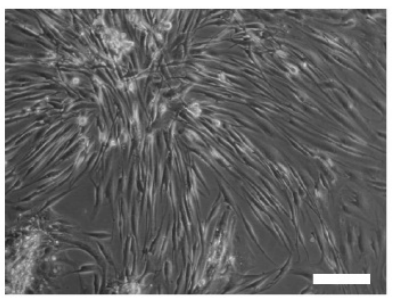

B
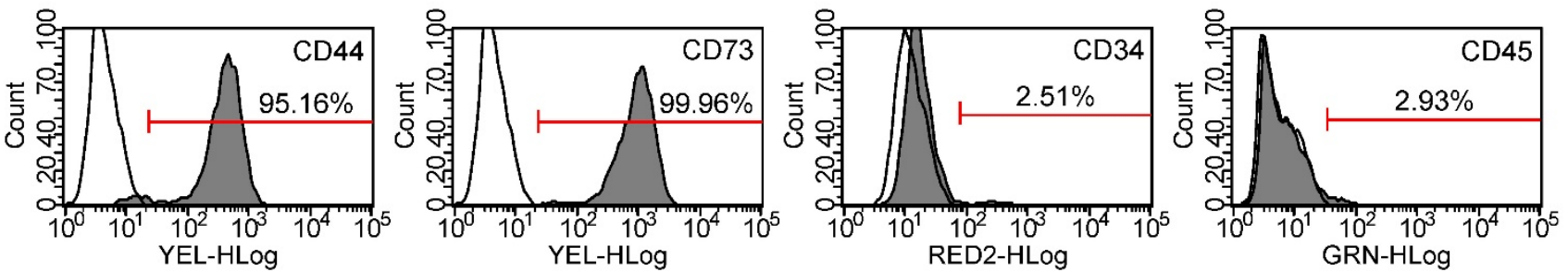

C
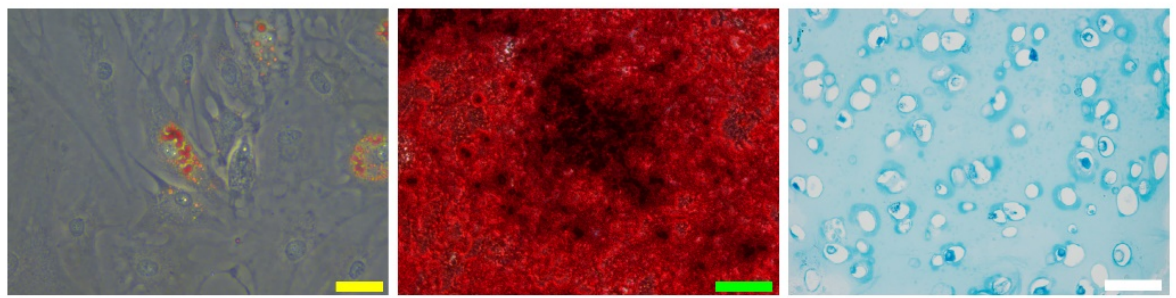

Figure 1. Isolation of human synovial-derived mesenchymal stem cells (SMSCs) from human synovial membrane. (A) SMSCs exhibited a typical spindle-shaped

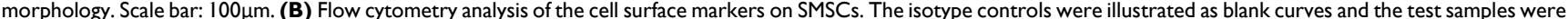
illustrated as solid gray curves. (C) SMSCs displayed ability of adipogenic differentiation (Yellow scale bar: $20 \mu \mathrm{m}$ ), osteogenic differentiation (Green scale bar: $100 \mu \mathrm{m})$ and chondrogenic differentiation ((white scale bar: $100 \mu \mathrm{m})$.

A

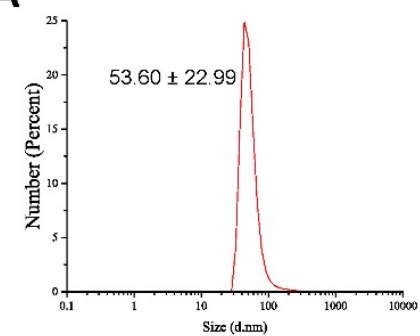

B

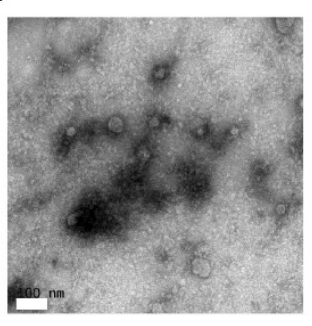

C

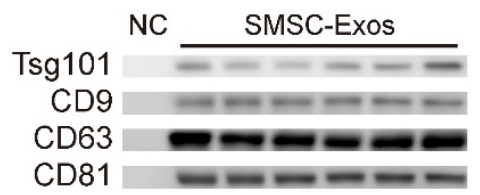

Figure 2. Characterization of exosomes released by human synovial-derived mesenchymal stem cells (SMSC-Exos). (A) Particle size distribution of SMSC-Exos measured by DLS. (B) Morphology of SMSC-Exos under a transmission electron microscopy. Scale bar: 100nm. (C) Western blotting analysis of exosomal surface markers (including CD9, CD63, CD81, and TSG 101). Exosomes from SMSCs of six patients were used. The ultrafiltration liquid of MesenGro hMSC Medium was used as negative control (NC). 
A

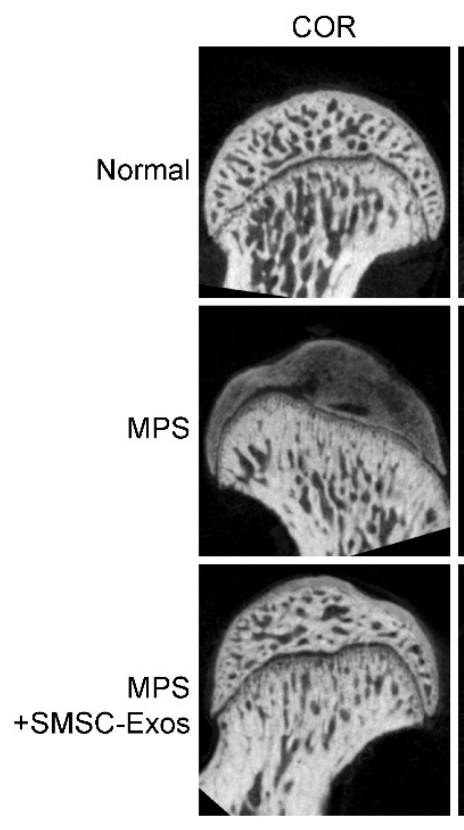

C

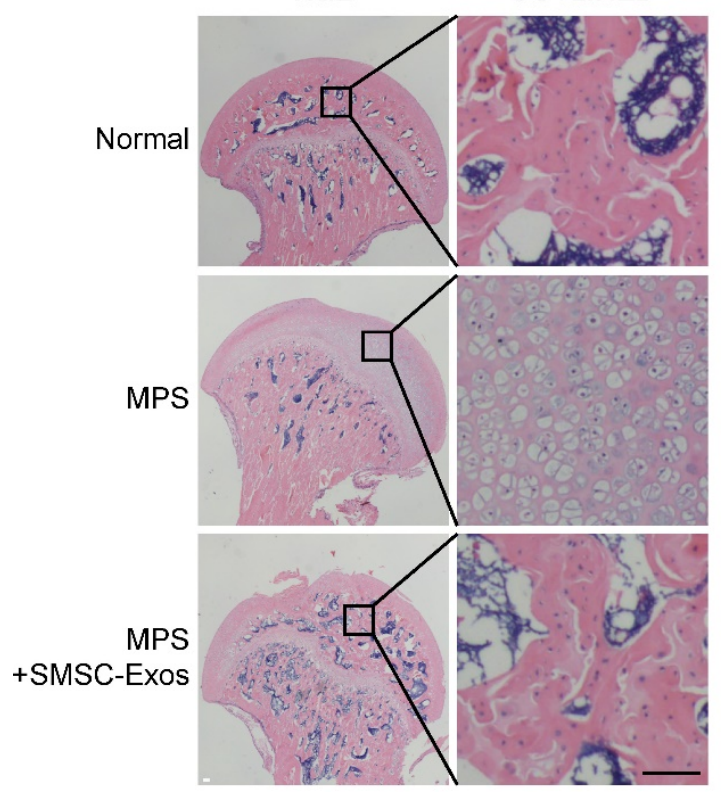

B

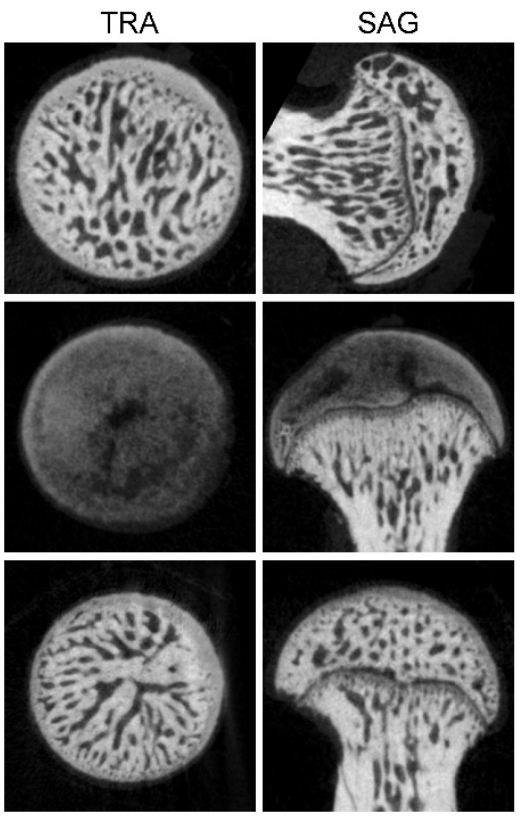

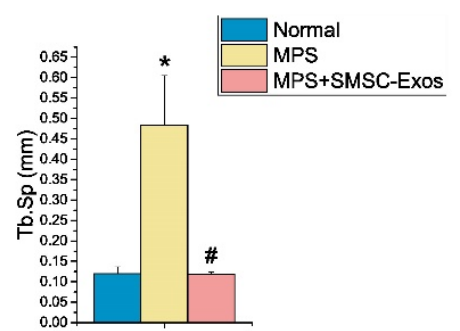
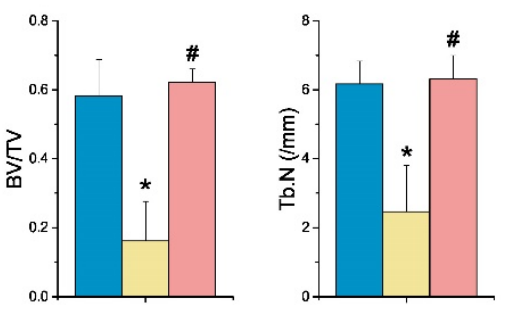

Figure 3. SMSC-Exos transplantation induces bone tissue-protective effects in GC-treated rats. (A) Reconstruction of coronal, transverse, and sagittal images of bones within the femoral heads in normal, methylprednisolone (MPS)-treated, and MPS+SMSC-Exos co-treated rats. (B) Quantitative analyses of the trabecular thickness (Tb.Th), trabecular separation (Tb.Sp), bone volume per tissue volume (BV/TV), and trabecular number (Tb.N) in different treatment groups. (C) H\&E staining of the femoral heads in rats receiving different treatments. Scale bar: $100 \mu \mathrm{m}$. (D) Immunohistochemical staining of OCN of the specimen in different treatment groups. Scale bar: $100 \mu m$.

\subsection{Protective effects of SMSC-Exos in the rat model of GC-induced ONFH}

To investigate the effects of SMSC-Exos on GC-induced ONFH, the rat model of ONFH was induced by intramuscularly injection of MPS, followed by intravenously administration of SMSC-Exos or an equal volume PBS. Six weeks after corresponding treatment, micro-CT scanning was carried out to quantitatively and qualitatively evaluate the bone tissues within the femoral head (Fig. 3A). The results showed $80 \%$ rats treated by multiple high-dose MPS exhibited significant trabecular changes, such as bone mineral loss and cystic degeneration in subchondral area of the femoral heads, as compared with the normal rats. On the contrary, the subchondral trabeculae of the femoral heads appeared intact and well distributed in rats after SMSC-Exos treatment, and only 20\% rats were found with very mild osteonecrotic changes. 
Quantitative analyses of all the micro-CT parameters then further confirmed the preventive efficacy of SMSC-Exos on GC-induced ONFH in rats. As shown in Fig. 3B, the microstructural parameters including Tb.Th, BV/TV, and Tb.N in MPS group $(0.075 \pm 0.015$, $16.21 \pm 11.38 \%$, and $2.46 \pm 1.34$, respectively) were markedly reduced compared with the normal group $(0.117 \pm 0.015, \quad 58.12 \pm 10.61 \%$, and $6.17 \pm 0.66$, respectively) $(P<0.05)$, but an additional treatment with SMSC-Exos $(0.121 \pm 0.010,62.19 \pm 3.89 \%$, and $6.31 \pm 0.68$, respectively; $P<0.05$ vs. MPS group) could remarkably reverse the MPS-induced reduction of these parameters. Moreover, the parameter Tb.Sp was significantly increased in MPS group than that in the normal group $(0.483 \pm 0.122$ vs. $0.119 \pm 0.017 ; P<0.05)$, and this increase could be profoundly inhibited by SMSC-Exos $(0.118 \pm 0.006 ; P<0.05$ vs. MPS group).

In consistent with the above findings, histological evidence based on H\&E staining (Fig. 3C) revealed that osteonecrosis in MPS group (model group) was obvious. In these MPS-treated rats, the trabecular bone of the femoral head became sparser and even disappeared, which were replaced by abnormal morphology. In contrast, the trabecular bone in the rats additionally treated with SMSC-Exos was well organized, and few trabecular structures and bone marrow were replaced by necrotic tissues. The incidence of histological ONFH in SMSC-Exos group $(20 \%, 3 / 15)$ was remarkably lower than that in MPS group $(80 \%, 12 / 15)(P<0.05)$, and no osteonecrosis

A
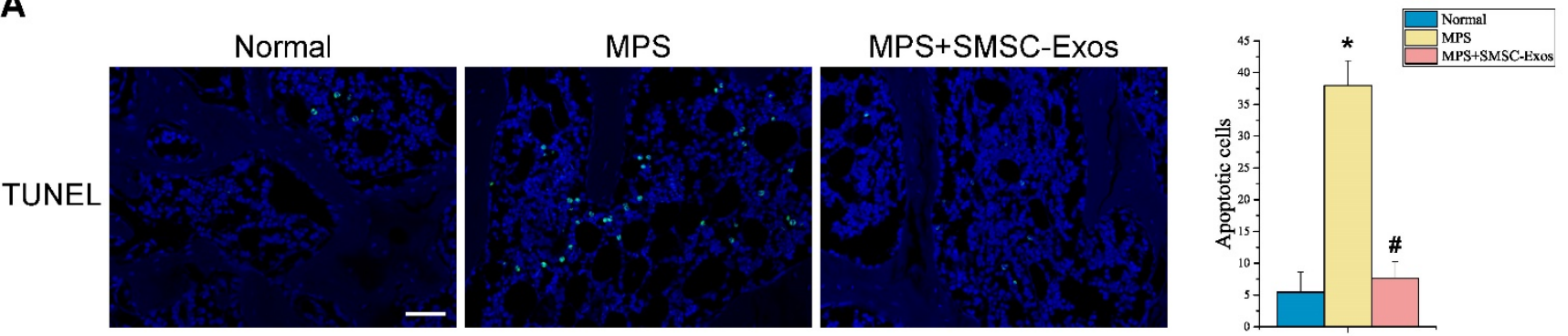

B
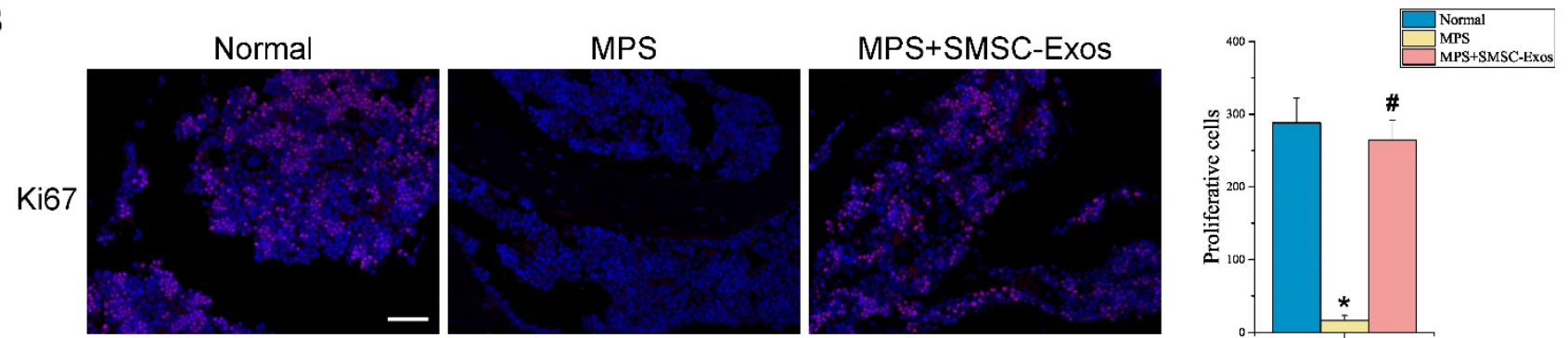

Figure 4. SMSC-Exos transplantation induces bone marrow cell-protective effects in GC-treated rats. (A) Cellular apoptosis in different treatment groups was analyzed by TUNEL assay. Scale bar: $50 \mu \mathrm{m}$. Quantitative analysis of the number of total apoptotic cells in (A). (B) The proliferation of cells in different treatment groups was analyzed ki67 immunostaining. Scale bar: $50 \mu \mathrm{m}$. Quantitative analysis of the number of total proliferative cells in (B). ( $* P<0.05$ compared with the normal rats (control), $\# P<0.05$ compared with the MPS-treated rats (model group).) 


\subsection{The uptake of SMSC-Exos by BMSCs}

We assessed the in vitro effects of SMSC-Exos on anti-apoptotic activities of BMSCs. Firstly, we determined whether SMSC-Exos could be internalized into BMSCs. We used a red fluorescent lipophilic dye (DiL) to label SMSCs and harvested their secreted exosomes, which were labeled by DiL upon the fusion of multivesicular body with the cell plasma membrane. After incubation with BMSCs for two hours, the DiL-labeled exosomes were transferred to the perinuclear region of BMSCs (Fig. 5A), indicating that SMSC-Exos were incorporated into BMSCs.

\section{4. promotion of cell proliferation and anti-apoptotic effects of SMSC-Exos on BMSCs}

To explore the functional roles of SMSC-Exos in cell proliferation and apoptosis under normal or high GC level conditions, BMSCs were cultured in a-MEM media supplemented with exosomes or/and DEX for a series of functional assays. The proliferation of BMSCs was examined by CCK-8 analysis (Fig. 5B). The results revealed that DEX treatment markedly reduced the proliferative capability of BMSCs, whereas incubation with SMSC-Exos resulted in a remarkable increase in BMSCs' proliferation. Moreover, the DEX-induced down-regulation of BMSCs' proliferation could be significantly inhibited by co-treatment with SMSC-Exos.

The apoptosis of BMSCs was assessed by flow cytometry with annexin V-FITC/PI double staining and the early apoptosis rate of BMSCs was measured (Fig. 5C-D). The results showed that BMSCs' apoptosis in DEX group was dramatically enhanced than that in the controls $(22.32 \pm 1.88 \%$ vs. $7.85 \pm 0.78 \%$; $P<0.05)$, whereas SMSC-Exos treatment $(1.93 \pm 0.52 \%$; $P<0.05$ vs. control group) markedly reduced the apoptosis of BMSCs and inhibited the DEX-induced apoptotic effect on BMSCs $(9.56 \pm 1.87 \%$ vs. $22.32 \pm 1.88 \% ; P<0.05)$.

\section{Discussion}

GC-associated ONFH is one of the most serious complications in patients who have received corticosteroids [12]. Although numerous therapeutic attempts have been made to halt the progression of GC-induced ONFH, the optimal treatment strategies are still being developed. In this study, we provided the first demonstration that early treatment with SMSC-Exos, the biomolecules released from SMSCs, could enhance bone marrow cells proliferation and prevent GC-induced ONFH in the rat model. We also found SMSC-Exos could enhance the proliferation and anti-apoptotic activities of BMSCs. Further, GC-induced suppression of cell proliferation and cellular apoptosis, was rescued by SMSC-Exos in vitro.
A

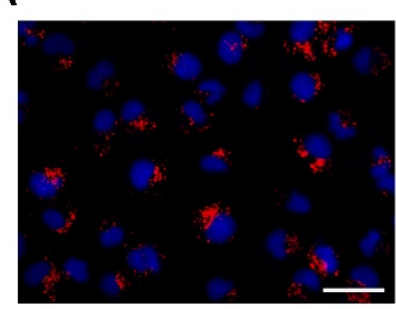

C

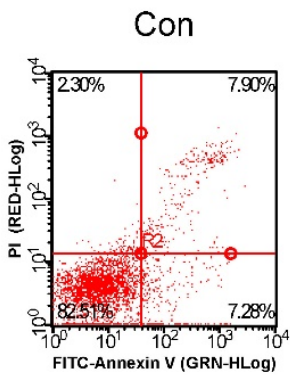

B

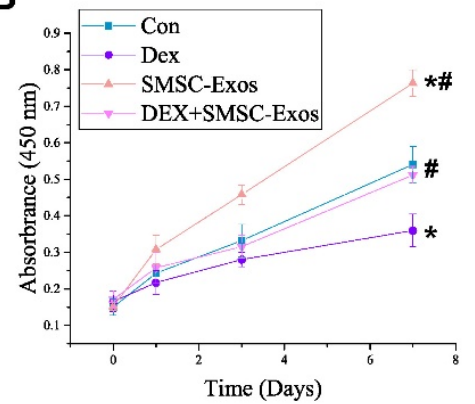

Figure 5. Uptake of SMSC-Exos by BMSCs and their proliferative and anti-apoptotic effects on endothelial cells. (A) Fluorescent microscopy analysis of DiL-labeled SMSC-Exos uptake by BMSCs. The red-labeled exosomes were visible in the perinuclear region of BMSCs. Scale bar: $50 \mu$ m. (B) The proliferation of BMSCs was analyzed by Cell Counting Kit-8 assay. (C) The apoptosis of BMSCs was assessed by flow cytometry with annexin V-FITC/propidium iodide (PI) double staining. Cells only stained with annexin V-FITC represent the early apoptotic cells. (D) Quantitative analysis of the early apoptosis rate of BMSCs. (*P< 0.05 compared with the control group, $\# P<0.05$ compared with the dexamethasone (DEX)-treated group). 
In the last decades, more and more studies have focused on the exosomes. Interestingly, it was reported that exosomes could promote proliferation and inhibit apoptosis by promoting $\beta$-catenin nuclear translocation, which would be inhibited by GC [24, 25], through carried proteins [26]. In addition, mRNAs [27], microRNAs [28, 29] and long non-coding RNAs [30] were recently found playing important roles in exosomes. The components of SMSC-Exos and the function of each component need to deepen in further research.

Autologous or allogenic osteogenic progenitor cells were generally used in cytotherapy researches. The injection of autologous MSCs combined with standard core decompression was firstly described in 1993[31]. Two main functions of MSCs were distinguished, the secretory function and participation in functional tissue restoration [32]. The clinical results are satisfactory in hip score, collapse, the need for THA and reduction of painful joint symptoms [31, 33-38].

However, the core decompression was a kind of invasive surgery for early stage of ONFH. And autologous MSCs injection can result in treatment failure attributed to GC-induced decreased activity of MSCs [39]. Exosomes can be used as intravenous injecting preventive medicine or even orally administered drugs [40,41], before, during and after using high-dose glucocorticoid. Since exosomes has no obviously adverse effect caused by immunogenicity [20,21], the application of allogeneic or even cross-species [41] exosomes could overcome difficulties caused by the decreased activity of autologous cells or the lack of cell resources.

MSCs were firstly described in human bone marrow $[42,43]$, called BMSCs which are promising for regenerative medicine. Later studies indicated that MSCs could be isolated from many various sources including adipose tissue, synovial membrane and umbilical cord besides bone marrow.

MSCs from older donors usually showed less proliferative ability and osteogenic potency [44-46]. The common sources of human BMSCs were patients received THA, amputation or surgery for obtaining autogenous cancellous bone. Patients received THA are usually in old age and isolated BMSCs showed low proliferative ability as expected. Young healthy males, getting amputation surgery caused by severe trauma, are ideal sources to obtain BMSCs of high quality. However, with improvements in healthcare and rising living standards, the number of young patients needed amputation will become scarce. BMSCs from autogenous cancellous bone is likely to be influenced by GC treatment. But SMSCs are easy to obtain from relatively young males in arthroscopic surgery for sports injuries.

SMSCs are pluripotent cells that can promote tissue repair. However, the direct application of culture-expanded cells is still hampered by many adverse effects [16-18]. Currently, a number of studies have indicated that the long-term functional benefits of transplanted cells on tissue repair are mainly mediated by a paracrine mechanism [47-49] and exosomes strongly contribute to the transplanted cells' paracrine effects [19]. There are multiple benefits in transplanting exosomes for therapeutic use [19-21]. As compared with the exogenously administrated cells, exosomes have no risk of donor-derived tumor development, as they lack machinery for replication. These nanoparticles are easily to reach the wound sites and have no vascular obstructive concern, because of their small size. Moreover, exosomes have a role in the suppression of allograft rejection. These features make exosomes an ideal candidate for tissue regenerative medicine.

Previous studies have shown that the administration of exosomes alone is sufficient to trigger the repairing process in various disease models, including limb ischemia [50], myocardial infarction [51], muscle injury [52], etc. However, so far, it remains unclear whether exosomes can exert beneficial effects on GC-induced ONFH. Herein, we demonstrated that SMSC-Exos transplantation could effectively prevent the GC-induced $\mathrm{ONFH}$ in rat models. Based on the histological results, we found that the infusion of SMSC-Exos decreased the GC-induced trabecular bones loss, bone marrow necrosis, and fatty cells accumulation. Results of TUNEL assay and ki67 staining showed that SMSC-Exos could reverse the GC-induced pro-apoptotic and anti-proliferative effects at the early phase. By using micro-CT analyses, we found that SMSC-Exos could improve bone mineral density, and the microstructures of trabecular bones in GC-induced ONFH rats. OCN staining further confirmed that SMSC-Exos enhanced the number of osteogenic cells in the femoral heads. We then evaluated the effects of SMSC-Exos on the behavior of BMSCs in vitro and found that SMSC-Exos could reverse the GC-induced anti-proliferation effect. We also determined whether SMSC-Exos could protect the BMSCs from the GC-induced apoptosis. Results showed that SMSC-Exos markedly inhibited the apoptosis of BMSCs induced by GC and serum deprivation.

\section{Conclusion}

In summary, we firstly demonstrated that SMSC-Exos could promote cell proliferation and prevent GC-induced ONFH in the rat model. We also 
found that SMSC-Exos could enhance the proliferation and anti-apoptotic responses of BMSCs in vitro, suggesting that the underlying mechanism by which SMSC-Exos enhance proliferation and anti-apoptosis effect of bone marrow cells may be the activation of BMSCs function. Further studies are required to assess the exact mechanism.

\section{Abbreviations}

ONFH: osteonecrosis of the femoral head

GC: glucocorticoid

SMSCs: synovial-derived mesenchymal stems

SMSC-Exos: exosomes derived from SMSCs

H\&E: Hematoxylin and eosin

OCN: osteocalcin

TUNEL: Terminal deoxynucleotidyl transferase

dUTP nick end labeling

BMSCs: bone marrow derived stromal cells

BM: bone marrow

MVBs: multivesicular bodies

PS: penicillin-streptomycin solution

DPBS: Dulbecco's Phosphate Buffered Saline

DMEM: Dulbecco's modified Eagle's medium

FBS: fetal bovine serum

BSA: bull serum albumin

DLS: Dynamic light scattering

TEM: transmission electron microscopy

Tb.Th: trabecular thickness

Tb.Sp: trabecular separation

BV/TV: bone volume per tissue volume

Tb.N: trabecular number

EDTA: ethylene diamine tetraacetic acid

IHC: immunohistochemical

a-MEM: a-Modified Eagle Medium

DEX: dexamethasone

CCK-8: cell counting kit-8

PI: propidium iodide

HRP: horseradish peroxidase

MPS: methylprednisolone

CT: computed tomogrphy

\section{Acknowledgements}

We thank the National Natural Science Foundation of China (Grant No.81301589 and No.81472066). This work was also supported by Joint Project Funding for Major Diseases in Shanghai (Grant No.2014ZYJB0301).

\section{Ethics}

This study was in adherence with the Declaration of Helsinki. All experimental and animal care procedures were approved by Animal Research Ethics Committee of Shanghai Sixth People's Hospital and performed in accordance with the guidelines of the National Institutes of Health Guidelines for the
Care and Use of Laboratory Animals. Written informed consent was obtained from each donor with the permission of the Institutional Review Board at Shanghai Sixth People's Hospital.

\section{Author Contributions}

S.C.G. participated in design of the study, performed the statistical analysis and drafted the manuscript. S.C.T. performed the animal experiment, helped to draft the manuscript and participated in design of the study and performed the statistical analysis. W.J.Y. helped the animal experiment. X.Q. participated the statistical analysis. J.G.S. participated in design of the study. C.Q.Z. conceived of the study and participated in its design. All authors read and approved the final manuscript.

\section{Conflict of interest}

The authors have declared that there is no conflict of interest.

\section{References}

1. Murata M, Kumagai K, Miyata N, Osaki M, Shindo H. Osteonecrosis in stroke-prone spontaneously hypertensive rats: effect of glucocorticoid. Journal of orthopaedic science : official journal of the Japanese Orthopaedic Association. 2007: 12: 289-95.

2. Kuribayashi M, Fujioka M, Takahashi KA, Arai Y, Hirata T, Nakajima S, et al. Combination analysis of three polymorphisms for predicting the risk for steroid-induced osteonecrosis of the femoral head. Journal of orthopaedic science : official journal of the Japanese Orthopaedic Association. 2008; 13: 297-303.

3. Chan KL, Mok CC. Glucocorticoid-induced avascular bone necrosis: diagnosis and management. The open orthopaedics journal. 2012; 6: 449-57.

4. Weinstein RS, Jilka RL, Parfitt AM, Manolagas SC. Inhibition of osteoblastogenesis and promotion of apoptosis of osteoblasts and osteocytes by glucocorticoids. Potential mechanisms of their deleterious effects on bone. The Journal of clinical investigation. 1998; 102: 274-82.

5. Wang X, Liu Y, Wang X, Liu R, Li J, Zhang G, et al. The Role of (99m)Tc-Annexin V Apoptosis Scintigraphy in Visualizing Early Stage Glucocorticoid-Induced Femoral Head Osteonecrosis in the Rabbit. BioMed research international. 2016; 2016: 7067259.

6. Guo S, Mao L, Ji F, Wang S, Xie Y, Fei H, et al. Activating AMP-activated protein kinase by an alpha1 selective activator compound 13 attenuates dexamethasone-induced osteoblast cell death. Biochemical and biophysical research communications. 2016; 471: 545-52.

7. Guo S, Xie Y, Fan JB, Ji F, Wang S, Fei H. alpha-Melanocyte stimulating hormone attenuates dexamethasone-induced osteoblast damages through activating melanocortin receptor 4-SphK1 signaling. Biochemical and biophysical research communications. 2016; 469: 281-7.

8. Zhang C, Zou YL, Ma J, Dang XQ, Wang KZ. Apoptosis associated with Wnt/beta-catenin pathway leads to steroid-induced avascular necrosis of femoral head. BMC musculoskeletal disorders. 2015; 16: 132.

9. Yu QS, Guo WS, Cheng LM, Lu YF, Shen JY, Li P. Glucocorticoids Significantly Influence the Transcriptome of Bone Microvascular Endothelial Cells of Human Femoral Head. Chinese medical journal. 2015; 128: 1956-63.

10. Ogata K, Katagiri W, Osugi M, Kawai T, Sugimura Y, Hibi H, et al. Evaluation of the therapeutic effects of conditioned media from mesenchymal stem cells in a rat bisphosphonate-related osteonecrosis of the jaw-like model. Bone. 2015; 74: 95-105

11. Terayama H, Ishikawa M, Yasunaga Y, Yamasaki T, Hamaki T, Asahara T, et al. Prevention of osteonecrosis by intravenous administration of human peripheral blood-derived CD34-positive cells in a rat osteonecrosis model. Journal of tissue engineering and regenerative medicine. 2011; 5: 32-40.

12. Sun $Y$, Feng $Y$, Zhang $C$, Cheng $X$, Chen $S, A i Z$ et al. Beneficial effect of autologous transplantation of endothelial progenitor cells on steroid-induced femoral head osteonecrosis in rabbits. Cell transplantation. 2011; 20: 233-43.

13. Zuk PA, Zhu M, Ashijian P, De Ugarte DA, Huang JI, Mizuno H, et al. Human adipose tissue is a source of multipotent stem cells. Molecular biology of the cell. 2002; 13: 4279-95.

14. Pittenger MF, Mackay AM, Beck SC, Jaiswal RK, Douglas R, Mosca JD, et al. Multilineage potential of adult human mesenchymal stem cells. Science (New York, NY). 1999; 284: 143-7. 
15. De Bari C, Dell'Accio F, Tylzanowski P, Luyten FP. Multipotent mesenchymal stem cells from adult human synovial membrane. Arthritis and rheumatism. 2001; 44: 1928-42.

16. Herberts CA, Kwa MS, Hermsen HP. Risk factors in the development of stem cell therapy. Journal of translational medicine. 2011; 9: 29.

17. Kansu E. Thrombosis in stem cell transplantation. Hematology. 2012; 17 Suppl 1: S159-62

18. Amariglio N, Hirshberg A, Scheithauer BW, Cohen Y, Loewenthal R, Trakhtenbrot L, et al. Donor-derived brain tumor following neural stem cell transplantation in an ataxia telangiectasia patient. PLoS medicine. 2009; 6: e1000029.

19. De Jong OG, Van Balkom BW, Schiffelers RM, Bouten CV, Verhaar MC. Extracellular vesicles: potential roles in regenerative medicine. Frontiers in immunology. 2014; 5: 608.

20. Burger D, Vinas JL, Akbari S, Dehak H, Knoll W, Gutsol A, et al. Human endothelial colony-forming cells protect against acute kidney injury: role of exosomes. The American journal of pathology. 2015; 185: 2309-23.

21. Xin H, Li Y, Chopp M. Exosomes/miRNAs as mediating cell-based therapy of stroke. Frontiers in cellular neuroscience. 2014; 8: 377.

22. Graneli C, Thorfve A, Ruetschi U, Brisby H, Thomsen P, Lindahl A, et al. Novel markers of osteogenic and adipogenic differentiation of human bone marrow stromal cells identified using a quantitative proteomics approach. Stem cell research. 2014; 12: 153-65.

23. Yang DH, Lee DW, Kwon YD, Kim HJ, Chun HJ, Jang JW, et al. Surface modification of titanium with hydroxyapatite-heparin-BMP-2 enhances the efficacy of bone formation and osseointegration in vitro and in vivo. Journal of tissue engineering and regenerative medicine. 2015; 9: 1067-77.

24. Zhou H, Mak W, Zheng Y, Dunstan CR, Seibel MJ. Osteoblasts directly control lineage commitment of mesenchymal progenitor cells through Wnt signaling. J Biol Chem. 2008; 283: 1936-45.

25. Yao W, Cheng Z, Busse C, Pham A, Nakamura MC, Lane NE. Glucocorticoid excess in mice results in early activation of osteoclastogenesis and adipogenesis and prolonged suppression of osteogenesis: a longitudinal study of gene expression in bone tissue from glucocorticoid-treated mice. Arthritis and rheumatism. 2008; 58: 1674-86.

26. Zhang B, Wang M, Gong A, Zhang X, Wu X, Zhu Y, et al. HucMSC-Exosome Mediated-Wnt4 Signaling Is Required for Cutaneous Wound Healing. Stem Cells. 2015; 33: 2158-68.

27. Gutkin A, Uziel O, Beery E, Nordenberg J, Pinchasi M, Goldvaser H, et al. Tumor cells derived exosomes contain hTERT mRNA and transform nonmalignant fibroblasts into telomerase positive cells. Oncotarget. 2016

28. Wang Y, Zhang L, Li Y, Chen L, Wang X, Guo W, et al. Exosomes/microvesicles from induced pluripotent stem cells deliver cardioprotective miRNAs and prevent cardiomyocyte apoptosis in the ischemic myocardium. Int J Cardiol. 2015; 192: 61-9.

29. Fang S, Xu C, Zhang Y, Xue C, Yang C, Bi H, et al. Umbilical Cord-Derived Mesenchymal Stem Cell-Derived Exosomal MicroRNAs Suppress Myofibroblast Differentiation by Inhibiting the Transforming Growth Factor-beta/SMAD2 Pathway During Wound Healing. Stem Cells Transl Med. 2016.

30. Qu L, Ding J, Chen C, Wu ZJ, Liu B, Gao Y, et al. Exosome-Transmitted IncARSR Promotes Sunitinib Resistance in Renal Cancer by Acting as a Competing Endogenous RNA. Cancer Cell. 2016; 29: 653-68.

31. Hernigou P, Beaujean F. Treatment of osteonecrosis with autologous bone marrow grafting. Clin Orthop Relat Res. 2002: 14-23.

32. Hernigou P, Trousselier M, Roubineau F, Bouthors C, Chevallier N, Rouard H, et al. Stem Cell Therapy for the Treatment of Hip Osteonecrosis: A 30-Year Review of Progress. Clin Orthop Surg. 2016; 8: 1-8.

33. Hernigou P, Poignard A, Zilber S, Rouard H. Cell therapy of hip osteonecrosis with autologous bone marrow grafting. Indian J Orthop. 2009; 43: 40-5.

34. Hernigou P, Lambotte JC. Volumetric analysis of osteonecrosis of the femur. Anatomical correlation using MRI. J Bone Joint Surg Br. 2001; 83: 672-5.

35. Hernigou P, Lambotte JC. Bilateral hip osteonecrosis: influence of hip size on outcome. Ann Rheum Dis. 2000; 59: 817-21.

36. Papakostidis C, Tosounidis TH, Jones E, Giannoudis PV. The role of "cell therapy" in osteonecrosis of the femoral head. A systematic review of the literature and meta-analysis of 7 studies. Acta Orthop. 2016; 87: 72-8.

37. Li X, Xu X, Wu W. Comparison of bone marrow mesenchymal stem cells and core decompression in treatment of osteonecrosis of the femoral head: a meta-analysis. Int J Clin Exp Pathol. 2014; 7: 5024-30.

38. Wang C, Wang Y, Meng HY, Yuan XL, Xu XL, Wang AY, et al. Application of bone marrow mesenchymal stem cells to the treatment of osteonecrosis of the femoral head. Int J Clin Exp Med. 2015; 8: 3127-35.

39. Houdek MT, Wyles CC, Packard BD, Terzic A, Behfar A, Sierra RJ. Decreased Osteogenic Activity of Mesenchymal Stem Cells in Patients With Corticosteroid-Induced Osteonecrosis of the Femoral Head. J Arthroplasty. 2016; 31: 893-8.

40. Arntz OJ, Pieters BC, Oliveira MC, Broeren MG, Bennink MB, de Vries M, et al. Oral administration of bovine milk derived extracellular vesicles attenuates arthritis in two mouse models. Mol Nutr Food Res. 2015; 59: 1701-12.

41. Munagala R, Aqil F, Jeyabalan J, Gupta RC. Bovine milk-derived exosomes for drug delivery. Cancer Lett. 2016; 371: 48-61.

42. Friedenstein AJ, Deriglasova UF, Kulagina NN, Panasuk AF, Rudakowa SF, Luria EA, et al. Precursors for fibroblasts in different populations of hematopoietic cells as detected by the in vitro colony assay method. Exp Hematol. 1974; 2: 83-92

43. Prockop DJ. Marrow stromal cells as stem cells for nonhematopoietic tissues. Science (New York, NY). 1997; 276: 71-4.

44. Aksu AE, Rubin JP, Dudas JR, Marra KG. Role of gender and anatomical region on induction of osteogenic differentiation of human adipose-derived stem cells. Ann Plast Surg. 2008; 60: 306-22.

45. Payne KA, Didiano DM, Chu CR. Donor sex and age influence the chondrogenic potential of human femoral bone marrow stem cells. Osteoarthritis Cartilage. 2010; 18: 705-13.

46. Choudhery MS, Badowski M, Muise A, Pierce J, Harris DT. Donor age negatively impacts adipose tissue-derived mesenchymal stem cell expansion and differentiation. Journal of translational medicine. 2014; $12: 8$

47. Bae SH, Kong TH, Lee HS, Kim KS, Hong KS, Chopp M, et al. Long-lasting paracrine effects of human cord blood cells on damaged neocortex in an animal model of cerebral palsy. Cell transplantation. 2012; 21: 2497-515.

48. Ratajczak MZ, Kucia M, Jadczyk T, Greco NJ, Wojakowski W, Tendera M, et al. Pivotal role of paracrine effects in stem cell therapies in regenerative medicine: can we translate stem cell-secreted paracrine factors and microvesicles into better therapeutic strategies? Leukemia. 2012; 26: 1166-73.

49. Wang CY, Yang HB, Hsu HS, Chen LL, Tsai CC, Tsai KS, et al. Mesenchymal stem cell-conditioned medium facilitates angiogenesis and fracture healing in diabetic rats. Journal of tissue engineering and regenerative medicine. 2012; 6: 559-69.

50. Hu GW, Li Q, Niu X, Hu B, Liu J, Zhou SM, et al. Exosomes secreted by human-induced pluripotent stem cell-derived mesenchymal stem cells attenuate limb ischemia by promoting angiogenesis in mice. Stem cell research \& therapy. 2015; 6: 10 .

51. Chen L, Wang Y, Pan Y, Zhang L, Shen C, Qin G, et al. Cardiac progenitor-derived exosomes protect ischemic myocardium from acute ischemia/reperfusion injury. Biochemical and biophysical research communications. 2013; 431: 566-71.

52. Nakamura Y, Miyaki S, Ishitobi H, Matsuyama S, Nakasa T, Kamei N, et al. Mesenchymal-stem-cell-derived exosomes accelerate skeletal muscle regeneration. FEBS letters. 2015; 589: 1257-65. 